\title{
New Method for Determination of Surface Distribution of Elastic Properties by Surface Acoustic
} Waves.

Mokryy O., Koshovyy V.

Karpenko Physico-Mechanical Institute of the National Academy of Science of Ukraine

5, Naukova str. Lviv, Ukraine, e-mail: mokomo@Iviv.farlep.net

\begin{abstract}
A new method for measuring of surface distribution of elastic properties is presented. The determination of velocity of surface acoustic waves (SAW) is used for it. The method is based on the new stabilization scheme of Michelson interferometer. It allows to measure the velocity of SAW in region of sample less than the wavelength of SAW and may be used for SAW of the frequency area one or ten $\mathrm{MHz}$.

The proposed method was analyzed by means of a numerical modeling method. The one-dimensional space distribution of non homogeneous elastic properties was investigated by this measurement scheme. This method may be used as non contact method under condition of using laser excitation of SAW.
\end{abstract}

\section{Introduction}

SAW are used for determination of the space distribution of the elastic properties of coated materials, composite structures, materials that have sustained surface modifications. These materials are important for the aircraft industry, medicine and other application.

The space distribution of an elastic properties measured by SAW is used for determination of space distribution of temperature [1], the investigation of material microstructure [2], visualization of defects [3]. The elastic properties are measured by acoustic waves. Methods of the measurement of the acoustic wave velocity is based on the measurement of travel time of acoustic waves between two points. That limits space resolution of these methods.

The laser ultrasound is a non contact method of excitation and detection of SAW and has a higher space resolution. The local SAW velocity was determined on titanium alloys by laser ultrasound [2]. The SAW frequency was $82 \mathrm{MHz}$ and the size of measurement region was $0.8 \mathrm{~mm}$. The local SAW velocity was measured by the acoustooptic diffraction [4]. This method is based on measurement of the angle of light diffraction and may be used for high frequency SAW. This method is difficult to use for SAW of the frequency area one or ten $\mathrm{MHz}$. But this range of frequency of SAW is usually used in NDT. We proposed the new method for measurement of the SAW local velocity by Michelson interferometer. The propose methods is simple and stabilization from the temperature drifts and vibrations. The methods allows us to measure the velocity of SAW in region of sample less than the wavelength of SAW.

\section{Principe of measurement of the SAW local velocity by Michelson interferometer}

The method of measurement of the local velocity is based on scheme detection of SAW by Michelson

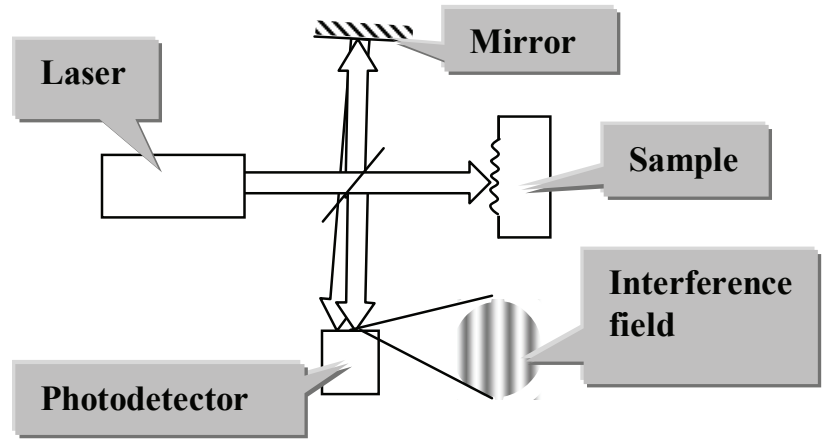

Fig.1 Scheme detection of SAW by Michelson interferometer interferometer $[5,6]$. The general scheme of the measurement setup is presented in fig.1. This setup differs from the typical setup of Michelson interferometer for detection of the SAW $[7,8]$. The difference consists in two things. The first one is that there is a certain angle between interfering beams. This angle appears as a result of the inclination of interferometer mirror (fig. 1). The presence of angle between interfering beams results in appearance of the spatial periodically modulated interference pattern. The second one is that optical beam width is bigger than

that usually used and is commensurate to wavelength of SAW.

Optical beams reflected from the sample and from the interferometer mirror are being interacted and an interference pattern is formed. The sample surface is shifted by the SAW. It causes the change of the 
length path difference. The change of intensity of interference field, which occurs if displacement associated with SAW propagation is being registered by a photodetector. This is a typical way to use Michelson interferometer for detecting SAW. The sensitivity of this scheme depends on the length path difference. The sensitivity of Michelson interferometer is equal to zero when the optical path difference is equal to $0.5 \lambda \mathrm{m}$, where $\lambda$ is the wavelength of optical radiation and $\mathrm{m}$ is an integer number.

The new stabilization scheme for detecting SAW by Michelson interferometer had been proposed in [5, 6]. The Michelson interferometer detects SAW and is stabilized against the change of length path difference when there is a certain angle between interfering beams as well as certain width of optical beam. The width of optical beam, when interferometer is stabilized is determined by the SAW wavelength. This fact is the basis of the method which is proposed for the measurement of the SAW velocity.

The process of measurement involves modulation of the length path difference by vibration of the interference mirror and change of the width of optical beam by the diaphragm. The photocurrent is modulated by the vibration of the interference mirror. The width of optical beam is selected with on the condition that the depth modulation of photocurrent is minimal. In this case it is possible to determine the wavelength of SAW. This method enables us to measure SAW wavelength, and local velocity is calculated from expression $\mathrm{V}=\Lambda \mathrm{f}$ where $\mathrm{f}$ is frequency of SAW.

\section{Numerical model of Michelson interferometer and numerical experiment}

In this paper we consider the case when the area of magnitude of a SAW wavelength is millimeters and amplitude of SAW is few nanometers. This conditions corresponds to conditions of using SAW in NDT.

For the analysis of performances of Michelson interferometer an approach of geometrical optics has been used [6]. A 1-D case was analyzed. The surface of the sample was taken as a mirror. An interference of optical beams with the same polarization and intensity was considered. In such a case an intensity of interference field is expressed by [9]:

$$
\mathrm{I}=\mathrm{I}_{1}+\mathrm{I}_{2}+2 \sqrt{\mathrm{I}_{1} \mathrm{I}_{2}} \cos \delta
$$

where $I_{1}$ and $I_{2}$ are the intensities of beams reflected from the mirror and from the sample, respectively, and $\delta$ is the phase difference between optical beams.

It was taken into account that optical beams had Gaussian distribution of intensity are given by:

$$
I_{1,2}=\frac{1}{\sqrt{2 \pi} \mathrm{a}} \mathrm{I}_{0} \mathrm{e}^{-\frac{\mathrm{x}^{2}}{2 \mathrm{a}^{2}}}
$$

Axis $\mathrm{x}$, is axis on the plane of photodetector.

The phase difference $\delta$ can appear as the result of different reasons and, in general, it is different in various points of the interference field.

First of all, $\delta$ is defined by the difference of distance to the mirror and sample $d$, the divergence angle of optical beams $\alpha$. Correspondingly, it is possible to write [10]:

$$
\delta_{1}=\frac{2 \pi}{\lambda}\left(2 \mathrm{~d}+2 \mathrm{~d} \sin ^{2} \alpha\right)
$$

The vibration of the mirror interferometer change of distance to the mirror and sample and $d$ is definite as:

$$
\mathrm{d}=\mathrm{d}_{0}+\mathrm{b} \sin (\Omega \mathrm{t})
$$

were $d_{0}$ is difference of distance to the mirror and sample at $t=0, b$ is amplitude and $\Omega$ is frequency of vibration of the interferometer mirror.

In case when interference beams made angle $\beta$ between them, the phase change between them is described by expression [9]:

$$
\delta_{2}=\frac{2 \pi}{\lambda} x \sin \beta
$$

Thus, the width of interference fringes is $L=N / \sin \beta$. 
We considered the case when the frequency range of SAW is a few $\mathrm{MHz}$ (wavelength is few millimeters) and its amplitude has a value of few of nanometers. As a result the reflected optical wave front is equal to the surface shape of the sample. The phase shift caused by the SAW, will take a value:

$$
\delta_{3}=\frac{2 \pi}{\lambda} 2 \mathrm{~h} \sin \left(\omega \mathrm{t}+\frac{2 \pi}{\Lambda} \mathrm{x}\right)
$$

where $\omega$ is the frequency of SAW and $\Lambda$ is the wavelength of SAW, $h$ is amplitude of SAW.

By taking into account the eqs.(1)-(6) the distribution of intensity in plane of interference pattern can be written as following:

$$
I=I_{1}+I_{2}+2 \sqrt{I_{1} I_{2}} \cos \left(\frac{2 \pi}{\lambda}\left(2\left(d_{0}+b \sin (\Omega t)\right)\left(1+\sin ^{2} \alpha\right)+x \sin \beta+2 h \sin \left(\omega t+\frac{2 \pi}{\Lambda} x\right)\right)\right)
$$

The photodetector is placed into the interference field (fig.1). The photocurrent is proportional to the total intensity of incident light on the photodetector.

$$
\mathrm{i}=\mathrm{g} \iint_{\mathrm{s}} \mathrm{I} \mathrm{d} x \mathrm{dy}
$$

$\mathrm{s}$ is the area of the interference field on photodetector, which was determined through diaphragm size, $g$ coefficient of proportionality.

The change of the magnitude of path length difference produce modulation of the photocurrent. The depth modulation is:

$$
\mathrm{G}=\frac{\Delta \mathrm{i}_{\max }-\Delta \mathrm{i}_{\min }}{\Delta \mathrm{i}_{\max }+\Delta \mathrm{i}_{\min }}
$$

$\Delta \mathrm{i}_{\max } \Delta \mathrm{i}_{\min }$ is maximum and minimum of $\mathrm{AC}$ amplitude of the photodetector current at the change of path length difference.

For numerical modeling the following values of parameters were used: $a=1.5 \mathrm{~mm}, \omega=6.28 \cdot 10^{6} \mathrm{~Hz}, \lambda=0.5$ $\mu \mathrm{m}, \alpha=0.002$. A displacement $\mathrm{h}$ of the surface of the sample under action of the SAW was taken sufficiently smaller than the optical wavelength and was accepted to $1 \mathrm{~nm}$. The position of minimum of

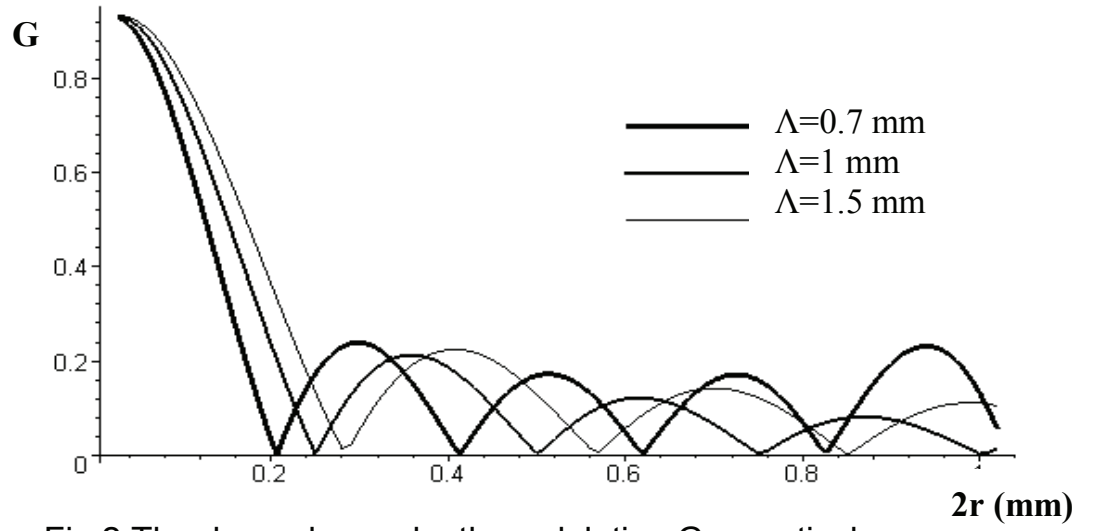

depth modulation $G$ is determined by the magnitude of wavelength of SAW the width of optical beam and the width of interference fringes. This dependence allows to determine the wavelength of SAW with help of measuring the width of optical beam under condition when the depth modulation of photocurrent is minimal. In this case the width of interference fringes is selected to be nearly equal to the wavelength of SAW. The magnitude of the wavelength of beam width $2 \mathrm{r}, \mathrm{L}=1 \mathrm{~mm}$

SAW is determined from measurement of width of the optical beam and the selected width of interference fringes. From these data the wavelength of SAW is calculated by numerical simulation.

\section{Experiment and result}

The scheme experimental setup is shown on fig.3-4. The geometry in which the interfering beams form some angle between themselves due to inclination of a mirror was used. On the surface of the sample the 


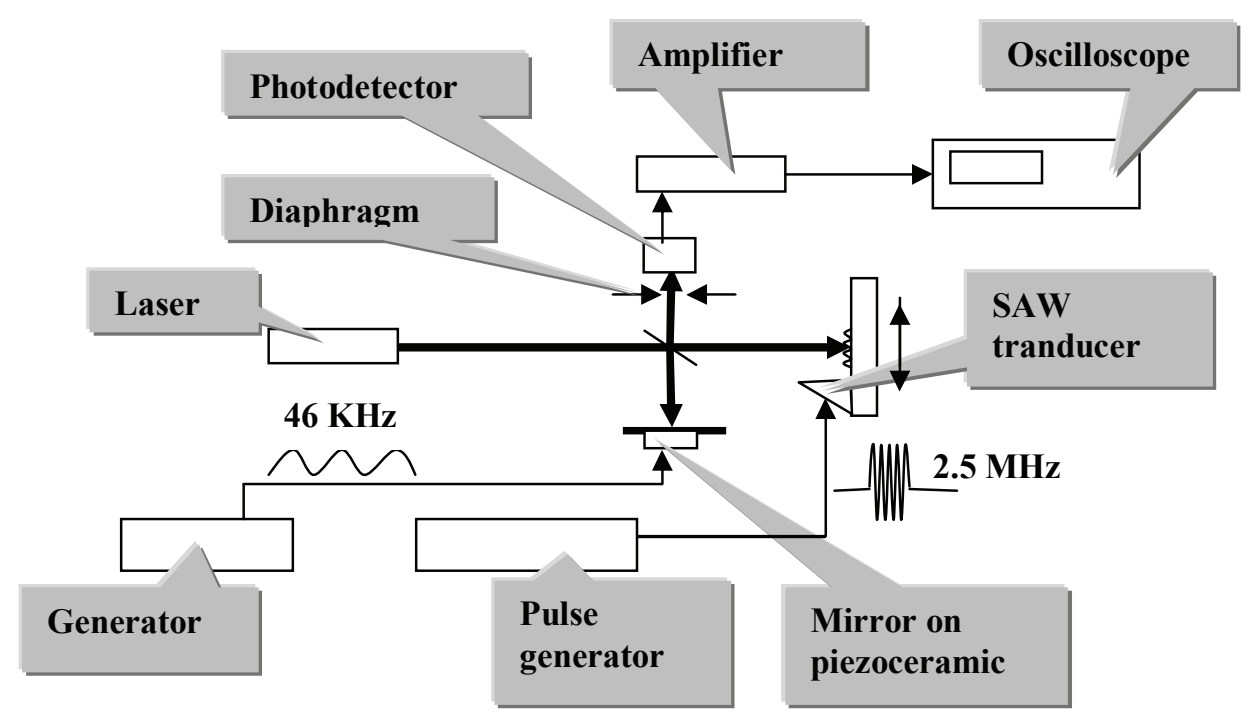

Fig. 3 Scheme of the experimental setup

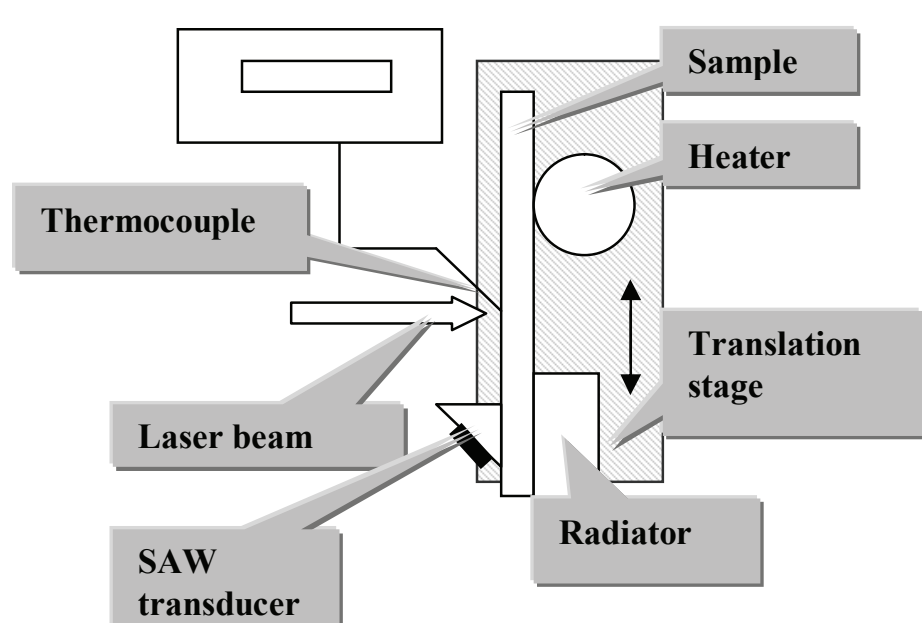

Fig.4 Scheme of disposal the sample and the heater

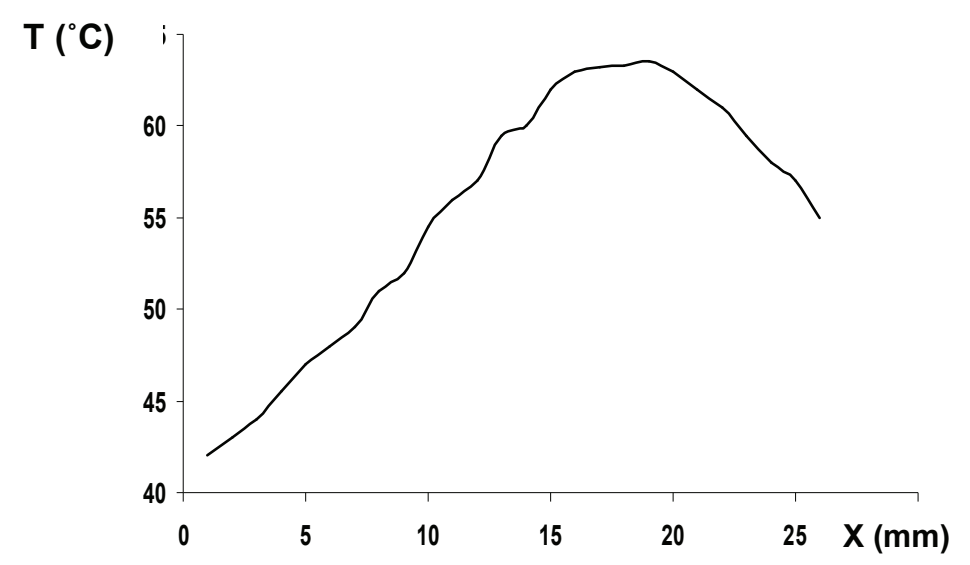

Fig.5 The space temperature distribution determined by thermocouple
SAW with frequency of $2.5 \mathrm{MHz}$ generated by prismatic piezoelectric transducer was propagated. The acoustic pulse had duration of $50-100$ $\mu \mathrm{s}$. The He-Ne laser with output radiation wavelength of $632.8 \mathrm{~nm}$ was used. The interferometer mirror had been fixed on a piezoelectric washer. Sinusoidal signal with frequency of 46 $\mathrm{KHz}$ was supplied to it for vibrating the mirror. This frequence is the resonance frequency of the piezoceramic. Under the action of this signal the mirror vibrated, which caused a change of path length difference. A signal which is supplied to the interferometer mirror and a pulse of the SAW are synchronized between themselves. An interference pattern is registered by the photodetector and the signal is observed on the oscilloscope. The setup also uses a diaphragm which allows changing of the width of the optic beam which falls onto the photodetector. The diaphragm is used for changing of the optical beam width. The optical beam width was selected under condition of minimum depth modulation. These data were used for the numerical calculation of the magnitude of SAW wavelength. But in the case that the of measuring the velocity change we used the selection of SAW frequency under the condition minimum depth modulation and optical beam width were invariable. This case is realized when the temperature dependence of the velocity or space distribution of the velocity are measured. The higher sensitivity is reached as a result of possibility to change the acoustic wave frequency more precisely than the diaphragm width. 
The fixing of the sample is presented in fig.4. The electrical heater is used to heat the sample. The temperature gradient is created in the sample. The temperature is measured by the thermocouple. The sample is fixed on the radiator for cooling. The temperature gradient of sample is created by the heater

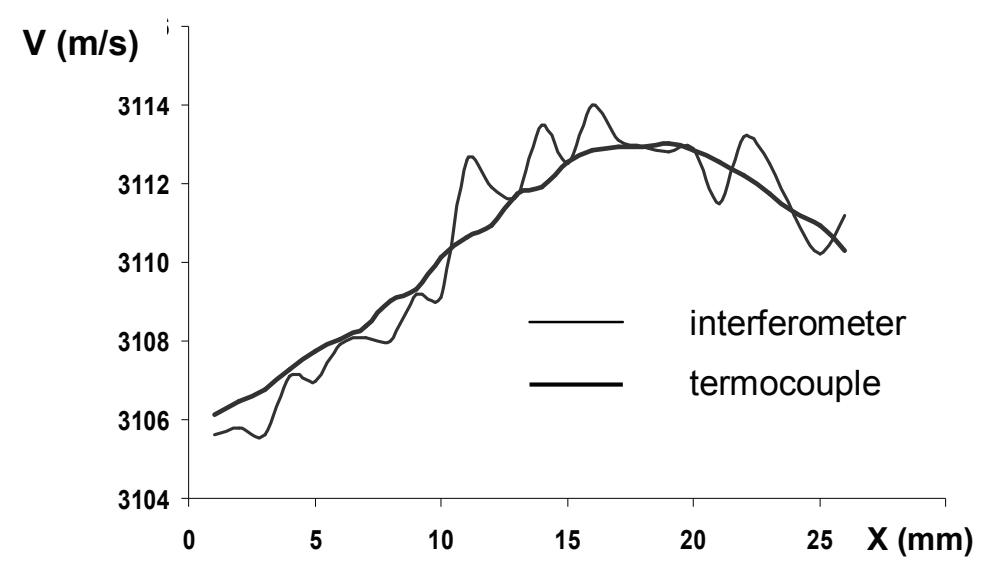

Fig. 6 The SAW velocity distribution determined by the interferometer and calculated by the temperature distribution and radiator. The sample together with the radiator and heater is fixed on translation stage. The translation stage can move relatively to the thermocouple and the beam of interferometer. This enables us to measure the space distribution of the temperature and SAW velocity.

Besides the temperature dependence of SAW velocity was measured by the pulse method. The contact piezoelectric transducers are used for this measurement. In this case the sample was heated homogenously. The glass plate with size $30 \times 60 \times 3$ $\mathrm{mm}^{3}$ is used as investigation sample. The sample surface was covered with a layer of metal for bigger reflection light. The measuring was made after the time relaxation of the sample temperature. The frequency of SAW wave was $2.5 \mathrm{MHz}$.

The results of measurement are shown in fig.5-6. The sample was heated to $70^{\circ} \mathrm{C}$. The measured magnitude of SAW velocity is $3100 \mathrm{~m} / \mathrm{s}$ and its temperature coefficient of it is $0.32 \mathrm{~m} /(\mathrm{s} \cdot \mathrm{C})$. The magnitude of SAW velocity measured by the pulse method and the velocity measured by proposed method differ by a few percent.

The data of measurement space distribution of temperature are used for calculation of space distribution of SAW velocity.

The temperature dependence of the SAW velocity was used for these calculation. The result of this calculation and SAW velocity dependence measured by interferometer is shown in fig. 6 . These data is correspond between themselves and it is demonstrated that the proposed method is suitable for measurement of the space distribution of SAW.

\section{Conclusion}

This paper presents a new method for measuring local SAW velocity based on Michelson interferometer. The new stabilization scheme of Michelson interferometer was applied. The numerical simulation this method was made. The one-dimension heterogeneous distribution of elastic properties as a result of onedimension heterogeneous distribution of temperature was investigated. It is shown that the proposed method is effective for determination of local elastic properties.

\section{References}

1. Ihara I., Takahashi M.:Laser-ultrasonic monitoring of temperature distribution of material surface during heating, Proceding of $1^{\text {st }}$ International Symposium on Laser Ultrasonics: Science, Technology and Applications, Monreal, Canada, July 16-18, 2008.

2. Sharples S.D., Clark M., Somekh M.G.: Spatially resolve acoustic spectroscopy for fast noncontact imaging of material microstructure, Optics express, Vol.14, No 22,10435-10440, 2006.

3. Focke O., Hildebrand A., Von Kopylow C.: Inspection of laser generated lanb waves using shearographic interferometry, Proceding of $1^{\text {st }}$ International Symposium on Laser Ultrasonics: Science, Technology and Applications, Monreal, Canada, July 16-18, 2008.

4. Oliver A.A. (edited) Acoustic Surface Waves, Springer-Verlag,Berlin-Heidelberg-NewYork,1978.

5. Mokryy O., Koshovyy V., Romanyshyn I., Sharamaga R.: New detection scheme of surface acoustic waves by Michelson interferometer, Proceeding of the topical meeting on optoinformatics 2008, St.Petersburg, Russia, 83-86, 2008.

6. Mokryy O., Koshovyy V., Romanyshyn I., Sharamaga R. Stabilized detection scheme of surface acoustic waves by Michelson interferometer.: Optica Aplicata.-Vol.XL, No 2, 449-458, 2010. 
7. Wagner J. W. Optical detection of ultrasound, in Physical acoustics: Ultrasonic Measurement Methods R.N. Thurston, Allan D.Pierce eds. V. XIX. Academic Press.Inc., Boston, SanDiego, New York, London, Sydney, Tokyo, Toronto, 201-265,1990.

8. Knuuttila J.V., Tikka P.T., Salomaa M. M.: Scanning Michelson interferometer for imading surface acoustic wave fields, Optics Letters, Vol. 25, Issue 9, 613-615, 2000.

9. Vest C. M.: Holographic interferometry, John Wiley \& Sons, New York, 1979.

10. Stone B. D.:Modeling interferometers with lens design software, Optical Engineering. Vol.39, 17481759, 2000. 